\title{
ON $\mathcal{J}$-CONTINUOUS FUNCTIONS
}

\author{
JaCEK Hejduk - Anna Loranty - Renata Wiertelak
}

ABSTRACT. This paper presents the properties of continuous functions equipped with the $\mathcal{J}$-density topology or natural topology in the domain and the range.

\section{Introduction}

Let $\mathbb{R}$ be the set of real numbers, $\mathbb{N}$ - the set of positive integers and $\mathcal{L}$ - the family of Lebesgue measurable sets in $\mathbb{R}$. By $\lambda(A)$ we shall denote the Lebesgue measure of $A \in \mathcal{L}$ and by $|I|$ - the length of an interval $I$. Furthermore, $\mathcal{T}_{\text {nat }}$ will denote the natural topology on $\mathbb{R}$ and $\mathcal{T}_{d}$ - the density topology on $\mathbb{R}$. Moreover, if $A, B \subset \mathbb{R}$ and $z \in \mathbb{R}$ then

$$
\begin{aligned}
A \triangle B & =(A \backslash B) \cup(B \backslash A), \\
A+z & =\{a+z: a \in A\}, \\
z A & =\{z \cdot a: a \in A\} .
\end{aligned}
$$

It is well known that a point $x_{0} \in \mathbb{R}$ is a density point of $A \in \mathcal{L}$ if

$$
\lim _{h \rightarrow 0^{+}} \frac{\lambda\left(A \cap\left[x_{0}-h, x_{0}+h\right]\right)}{2 h}=1 .
$$

An equivalent form of this condition is the following one:

$$
\lim _{\substack{h \rightarrow 0^{+}, h_{2} \rightarrow 0^{+} \\ h_{1}+h_{2}>0}} \frac{\lambda\left(A \cap\left[x_{0}-h_{1}, x_{0}+h_{2}\right]\right)}{h_{1}+h_{2}}=1 .
$$

Sometimes it is written in the form (see [6]):

$$
\forall\left\{J_{n}\right\}_{n \in \mathbb{N}}\left(0 \in \bigcap_{n \in \mathbb{N}} J_{n} \wedge\left|J_{n}\right| \underset{n \rightarrow \infty}{\longrightarrow} 0\right) \Rightarrow \lim _{n \rightarrow \infty} \frac{\lambda\left(A \cap\left(J_{n}+x_{0}\right)\right)}{\left|J_{n}\right|}=1,
$$

where $\left\{J_{n}\right\}_{n \in \mathbb{N}}$ is a sequence of non-degenerate closed intervals.

(C) 2016 Mathematical Institute, Slovak Academy of Sciences.

2010 Mathematics Subject Classification: 54A10, 26A15, 54A20.

Keywords: $\mathcal{J}$-density topologies, $\mathcal{J}$-approximately continuous functions. 
By $\mathcal{J}$ we will denote a sequence of non-degenerate and closed intervals $\left\{J_{n}\right\}_{n \in \mathbb{N}}$ tending to zero, that means $\operatorname{diam}\left\{J_{n} \cup\{0\}\right\} \underset{n \rightarrow \infty}{\longrightarrow} 0$. To shorten the notation, we will write $\mathcal{J}$ instead of $\left\{J_{n}\right\}_{n \in \mathbb{N}}$.

From now on, the family of all sequences of intervals tending to zero will be denoted by $\Im$. Moreover, we will consider sequences which differ in only a finite number of their terms to be identical.

Definition 1. Let $\mathcal{J} \in \Im$. We shall say that a point $x_{0} \in \mathbb{R}$ is a $\mathcal{J}$-density point of a set $A \in \mathcal{L}$ if

$$
\lim _{n \rightarrow \infty} \frac{\lambda\left(A \cap\left(x_{0}+J_{n}\right)\right)}{\left|J_{n}\right|}=1 .
$$

If $A \in \mathcal{L}$ and $\mathcal{J} \in \Im$, then

$$
\Phi_{\mathcal{J}}(A):=\{x \in \mathbb{R}: x \text { is a } \mathcal{J} \text {-density point of the set } A\} .
$$

Property 2 ([5]). If $A \in \mathcal{L}$ and $\mathcal{J} \in \Im$, then $\Phi_{\mathcal{J}}(A) \in \mathcal{L}$.

Moreover, this operator fulfills the following property.

Property 3 ([5]). For any sets $A, B \in \mathcal{L}$ and $\mathcal{J} \in \Im$ we have:

(1) $\Phi_{\mathcal{J}}(\emptyset)=\emptyset, \Phi_{\mathcal{J}}(\mathbb{R})=\mathbb{R}$

(2) $\lambda(A \triangle B)=0 \Rightarrow \Phi_{\mathcal{J}}(A)=\Phi_{\mathcal{J}}(B)$;

(3) $\Phi_{\mathcal{J}}(A \cap B)=\Phi_{\mathcal{J}}(A) \cap \Phi_{\mathcal{J}}(B)$.

It turned out that the analogue of the Lebesgue Density Theorem does not hold for every sequence of intervals $\mathcal{J}$ tending to zero. Studying paper [2], we can find a construction of a sequence $\mathcal{J} \in \Im$ and a set $A \in \mathcal{L}$ of positive measure such that $\lambda\left(\Phi_{\mathcal{J}}(A) \cap A\right)=0$. However, if we consider a subfamily $\Im_{\alpha} \subset \Im$ such that for any sequence $\mathcal{J} \in \Im_{\alpha}$ we have

$$
\alpha(\mathcal{J}):=\limsup _{n \rightarrow \infty} \frac{\operatorname{diam}\left(J_{n} \cup\{0\}\right)}{\left|J_{n}\right|}<\infty,
$$

then the analogue of the Lebesgue Density Theorem holds.

TheOREM 4 ([్[]). If $\mathcal{J} \in \Im_{\alpha}$ and $A \in \mathcal{L}$, then $\lambda\left(\Phi_{\mathcal{J}}(A) \triangle A\right)=0$.

Property 3 and Theorem 4 mean that an operator $\Phi_{\mathcal{J}}: \mathcal{L} \rightarrow \mathcal{L}$ is a lower density operator for every $\mathcal{J} \in \Im_{\alpha}$. Therefore the family

$$
\mathcal{T}_{\mathcal{J}}:=\left\{A \in \mathcal{L}: A \subset \Phi_{\mathcal{J}}(A)\right\}
$$

is a topology on $\mathbb{R}$ such that $\mathcal{T}_{d} \subset \mathcal{T}_{\mathcal{J}}$ (see [5]).

Obviously, one can ask what will happen if we consider any sequence $\mathcal{J} \in \Im$. In this case, we can prove the following fact.

TheOREm 5 ([5]). For every sequence $\mathcal{J} \in \Im$ and every set $A \in \mathcal{L}$ we have

$$
\lambda\left(\Phi_{\mathcal{J}}(A) \backslash A\right)=0 .
$$


By Property 3 and Theorem [5 we obtain that an operator $\Phi_{\mathcal{J}}: \mathcal{L} \rightarrow \mathcal{L}$ is an almost lower density operator. Theorem 10 in [5] says that the family

$$
\mathcal{T}_{\mathcal{J}}=\left\{A \in \mathcal{L}: A \subset \Phi_{\mathcal{J}}(A)\right\}
$$

is a topology on $\mathbb{R}$ such that $\mathcal{T}_{\text {nat }} \subset \mathcal{T}_{\mathcal{J}}$ and $\mathcal{T}_{\text {nat }} \neq \mathcal{T}_{\mathcal{J}}$.

It is easy to see that if $\mathcal{J}=\left\{\left[-\frac{1}{n}, \frac{1}{n}\right]\right\}_{n \in \mathbb{N}}$, then $x_{0}$ is a $\mathcal{J}$-density point of a set $A \in \mathcal{L}$ if and only if $x_{0}$ is a density point of $A$ (see [6]). Moreover, if we consider an unbounded and nondecreasing sequence $\langle s\rangle=\left\{s_{n}\right\}_{n \in \mathbb{N}}$ of positive numbers and a sequence $\mathcal{J}=\left\{\left[-\frac{1}{s_{n}}, \frac{1}{s_{n}}\right]\right\}_{n \in \mathbb{N}}$, then the notion of a $\mathcal{J}$-density point of a set $A \in \mathcal{L}$ is equivalent to the notion of an $\langle s\rangle$-density point of $A$ (see [3]).

From the definition of a $\mathcal{J}$-density point and a $\mathcal{J}$-density topology it is easy to conclude the following property.

Property 6. For every $\mathcal{J} \in \Im$ and every set $A \in \mathcal{L}$ the following properties hold:

(i) $\underset{x \in \mathbb{R}}{\forall} \underset{a \in \mathbb{R}}{\forall} x \in \Phi_{\mathcal{J}}(A) \Leftrightarrow(x+a) \in \Phi_{\mathcal{J}}(A+a)$,

(ii) $\underset{x \in \mathbb{R}}{\forall} \underset{m \in \mathbb{R} \backslash\{0\}}{\forall} x \in \Phi_{\mathcal{J}}(A) \Leftrightarrow m x \in \Phi_{m \mathcal{J}}(m A)$,

(iii) $\underset{a \in \mathbb{R}}{\forall} A \in \mathcal{T}_{\mathcal{J}} \Leftrightarrow(A+a) \in \mathcal{T}_{\mathcal{J}}$,

(iv) $\underset{m \in \mathbb{R} \backslash\{0\}}{\forall} A \in \mathcal{T}_{\mathcal{J}} \Leftrightarrow m A \in \mathcal{T}_{m \mathcal{J}}$.

Since for any $\mathcal{J} \in \Im$, the operator $\Phi_{\mathcal{J}}$ is an almost lower density operator, so, by [4, Theorem 25.27], we obtain immediately the following claim.

TheOREM 7. Let $\mathcal{J} \in \Im$.

(i) $\left(\mathbb{R}, \mathcal{T}_{\mathcal{J}}\right)$ is neither a first countable, nor a second countable, nor a separable, nor a Lindelöf space,

(ii) $\lambda(A)=0$ if and only if $A$ is a closed and discrete set with respect to a topology $\mathcal{T}_{\mathcal{J}}$

(iii) a set $A \subset \mathbb{R}$ is compact with respect to a topology $\mathcal{T}_{\mathcal{J}}$ if and only if $A$ is finite.

We say that a sequence of intervals $\mathcal{J}=\left\{\left[a_{n}, b_{n}\right]\right\}_{n \in \mathbb{N}} \in \Im$ is right-side (left-side) tending to zero if there exists $n_{0} \in \mathbb{N}$ such that $b_{n}>0\left(a_{n}<0\right)$ for $n \geq n_{0}$ and

$$
\lim _{n \rightarrow \infty} \frac{\min \left\{0, a_{n}\right\}}{b_{n}}=0 \quad\left(\lim _{n \rightarrow \infty} \frac{\max \left\{0, b_{n}\right\}}{a_{n}}=0\right) .
$$

Obviously, if for a sequence $\mathcal{J} \in \Im$ there exists $n_{0} \in \mathbb{N}$ such that $J_{n} \subset[0, \infty)$ $\left(J_{n} \subset(-\infty, 0]\right)$ for $n>n_{0}$, then $\mathcal{J}$ is right-side (left-side) tending to zero. 


\section{JACEK HEJDUK — ANNA LORANTY — RENATA WIERTELAK}

A sequence of intervals $\mathcal{J} \in \Im$ is one-side tending to zero if it is right-side or left-side tending to zero.

TheOREM 8. If $\mathcal{J}$ is a sequence of intervals tending to zero, then $[0, b) \in \mathcal{T}_{\mathcal{J}}$ for $b>0\left((a, 0] \in \mathcal{T}_{\mathcal{J}}\right.$ for $\left.a<0\right)$ if and only if the sequence $\mathcal{J}$ is right-side (left-side) tending to zero.

Proof. We give the proof only for the case when the sequence $\mathcal{J}$ is right-side tending to zero; the second case is left to the reader. Sufficiency. Let $\mathcal{J}=\left\{\left[a_{n}, b_{n}\right]\right\}_{n \in \mathbb{N}}$ be a sequence of intervals right-side tending to zero and $b>0$. Without the loss of generality we may assume that $0<b_{n}<b$ for $n \in \mathbb{N}$. It is sufficient to show that $0 \in \Phi_{\mathcal{J}}([0, b))$. We prove that for every increasing sequence $\left\{n_{k}\right\}_{k \in \mathbb{N}}$ there exists subsequence $\left\{n_{k_{m}}\right\}_{m \in \mathbb{N}}$ such that

$$
\lim _{m \rightarrow \infty} \frac{\lambda\left([0, b) \cap J_{n_{k_{m}}}\right)}{\left|J_{n_{k_{m}}}\right|}=1 .
$$

Let $\left\{n_{k}\right\}_{k \in \mathbb{N}}$ be an increasing sequence of natural numbers. If $a_{n_{k}} \geq 0$ for infinitely many $k \in \mathbb{N}$, then we choose subsequence $\left\{n_{k_{m}}\right\}_{m \in \mathbb{N}}$ such that $a_{n_{k_{m}}} \geq 0$ for $m \in \mathbb{N}$. Hence, $J_{n_{k_{m}}} \subset[0, b)$ for $m \in \mathbb{N}$. Therefore, condition (11) is fulfilled.

Now, we assume that $a_{n_{k}} \geq 0$ only for finite numbers $k \in \mathbb{N}$. Then, there is a $k_{1} \in \mathbb{N}$ such that for $k>k_{1}$ we have $a_{n_{k}}<0$ and

$$
\frac{\lambda\left([0, b) \cap J_{n_{k}}\right)}{\left|J_{n_{k}}\right|}=\frac{\left|J_{n_{k}}\right|-\lambda\left(\left[a_{n_{k}}, 0\right)\right)}{\left|J_{n_{k}}\right|}=1-\frac{-a_{n_{k}}}{\left|J_{n_{k}}\right|} \geq 1+\frac{a_{n_{k}}}{b_{n_{k}}} .
$$

The above and the assumption that the sequence $\mathcal{J}$ is right-side tending to zero implies condition (1).

We conclude that, in both cases, $0 \in \Phi_{\mathcal{J}}([0, b))$.

NeCESSITY. Let $\mathcal{J}=\left\{\left[a_{n}, b_{n}\right]\right\}_{n \in \mathbb{N}} \in \Im$ and $[0, b) \in \mathcal{T}_{\mathcal{J}}$, where $b>0$. Obviously, $0 \in \Phi_{\mathcal{J}}([0, b))$ and $b_{n} \leq 0$ only for finitely many $n \in \mathbb{N}$. Without loss of generality we may assume that $b_{n}>0$ for $n \in \mathbb{N}$. Suppose that $\mathcal{J}$ is not right-side tending to zero. Then,

$$
\limsup _{n \in \mathbb{N}} \frac{\left|\min \left\{0, a_{n}\right\}\right|}{b_{n}}=\beta>0 .
$$

So, there exists subsequence $\left\{n_{k}\right\}_{k \in \mathbb{N}}$ such that

$$
\lim _{k \rightarrow \infty} \frac{\left|\min \left\{0, a_{n_{k}}\right\}\right|}{b_{n_{k}}}=\beta
$$

and also that $a_{n_{k}}<0$ for $k \in \mathbb{N}$. Moreover, there exists $k_{0} \in \mathbb{N}$ such that

$$
\frac{1}{2} \beta b_{n_{k}}<\left|a_{n_{k}}\right|<\frac{3}{2} \beta b_{n_{k}} \quad \text { for } \quad k>k_{0} .
$$


Then,

$$
\begin{aligned}
\frac{\lambda\left([0, b) \cap J_{n_{k}}\right)}{\left|J_{n_{k}}\right|} & =\frac{\lambda\left(J_{n_{k}}\right)-\lambda\left(\left[a_{n_{k}}, 0\right)\right)}{\left|J_{n_{k}}\right|}=1-\frac{\left|a_{n_{k}}\right|}{b_{n_{k}}+\left|a_{n_{k}}\right|} \\
& \leq 1-\frac{1 / 2 \beta b_{n_{k}}}{b_{n_{k}}+3 / 2 \beta b_{n_{k}}}=1-\frac{\beta}{2+3 \beta} .
\end{aligned}
$$

It implies that $0 \notin \Phi_{\mathcal{J}}([0, b))$. This contradiction finishes the proof.

A direct consequence of the above theorem is

TheOREM 9. If a sequence of intervals $\mathcal{J}$ is tending to zero then $[a, b) \in \mathcal{T}_{\mathcal{J}}$ $\left((a, b] \in \mathcal{T}_{\mathcal{J}}\right)$ for $a<b$ if and only if the sequence $\mathcal{J}$ is right-side (left-side) tending to zero.

\section{Continuous functions}

For $\mathcal{J} \in \Im$ we consider four families of continuous functions defined as follows:

$$
\begin{aligned}
\mathcal{C}_{\text {nat, nat }} & =\left\{f:\left(\mathbb{R}, \mathcal{T}_{\text {nat }}\right) \rightarrow\left(\mathbb{R}, \mathcal{T}_{\text {nat }}\right) \text { and } f \text { is continuous }\right\}, \\
\mathcal{C}_{\text {nat, } \mathcal{J}} & =\left\{f:\left(\mathbb{R}, \mathcal{T}_{\text {nat }}\right) \rightarrow\left(\mathbb{R}, \mathcal{T}_{\mathcal{J}}\right) \text { and } f \text { is continuous }\right\}, \\
\mathcal{C}_{\mathcal{J}, \text { nat }} & =\left\{f:\left(\mathbb{R}, \mathcal{T}_{\mathcal{J}}\right) \rightarrow\left(\mathbb{R}, \mathcal{T}_{\text {nat }}\right) \text { and } f \text { is continuous }\right\}, \\
\mathcal{C}_{\mathcal{J}, \mathcal{J}} & =\left\{f:\left(\mathbb{R}, \mathcal{T}_{\mathcal{J}}\right) \rightarrow\left(\mathbb{R}, \mathcal{T}_{\mathcal{J}}\right) \text { and } f \text { is continuous }\right\} .
\end{aligned}
$$

Property 10. For $\mathcal{J} \in \Im$ the family $\mathcal{C}_{\text {nat, } \mathcal{J}}$ consists of constant functions.

Proof. Let $f \in \mathcal{C}_{n a t, \mathcal{J}}$ and $a, b \in \mathbb{R}$ be such that $a<b$. Then $f([a, b])$ is a nonempty and compact set with respect to the topology $\mathcal{T}_{\mathcal{J}}$. By Theorem 7 $f([a, b])$ is finite. Moreover, it is a connected set in $\mathcal{T}_{\text {nat }}$, so $f([a, b])$ is a singleton. Hence, $f(a)=f(b)$, and the function $f$ is constant.

Property 11. For $\mathcal{J} \in \Im$, the following inclusions hold:

(i) $\mathcal{C}_{\text {nat, } \mathcal{J}} \subsetneq \mathcal{C}_{\text {nat, nat }} \subset \mathcal{C}_{\mathcal{J}, \text { nat }}$,

(ii) $\mathcal{C}_{\text {nat, } \mathcal{J}} \subsetneq \mathcal{C}_{\mathcal{J}, \mathcal{J}} \subset \mathcal{C}_{\mathcal{J}, \text { nat }}$.

P r o o f. All the inclusions are the consequence of the fact that $\mathcal{T}_{\text {nat }} \subset \mathcal{T}_{\mathcal{J}}$. The inclusions $\mathcal{C}_{\text {nat, } \mathcal{J}} \subset \mathcal{C}_{\text {nat, nat }}$ and $\mathcal{C}_{\text {nat, } \mathcal{J}} \subset \mathcal{C}_{\mathcal{J}, \mathcal{J}}$ are proper because the identical function is a member of $\mathcal{C}_{\text {nat, nat }}$ and $\mathcal{C}_{\mathcal{J}, \mathcal{J}}$ but not $\mathcal{C}_{\text {nat, } \mathcal{J}}$.

Property 12. If $\mathcal{J}$ is a sequence of intervals one-side tending to zero then:

(i) $\mathcal{C}_{\text {nat, nat }} \backslash \mathcal{C}_{\mathcal{J}, \mathcal{J}} \neq \emptyset$,

(ii) $\mathcal{C}_{\mathcal{J}, \mathcal{J}} \backslash \mathcal{C}_{\text {nat }, \text { nat }} \neq \emptyset$. 
P r o of. We give the proof only for the case when the sequence $\mathcal{J}$ is right side-tending to zero; the second case is left to the reader. To show the first condition, we consider the function $f(x)=-x^{2}$. Obviously, $f \in \mathcal{C}_{\text {nat, nat }} \subset \mathcal{C}_{\mathcal{J} \text {, nat }}$. By Theorem 9, $[-1,1) \in \mathcal{T}_{\mathcal{J}}$ but $f^{-1}([-1,1))=[-1,1] \notin \mathcal{T}_{\mathcal{J}}$. Thus, $f \notin \mathcal{C}_{\mathcal{J}}, \mathcal{J}$, and condition (i) is proved.

To prove the second condition, we define the function

$$
h(x)=x-k \quad \text { for } \quad x \in[k, k+1), k \in \mathbb{Z} .
$$

It is easy to see that for every set $A \subset \mathbb{R}$

$$
h^{-1}(A)=\bigcup_{k \in \mathbb{Z}}((A \cap[0,1))+k)
$$

holds.

Thus for every set $A \in \mathcal{T}_{\mathcal{J}}$, by Theorem 9 and Property [6, we have that $h^{-1}(A) \in \mathcal{T}_{\mathcal{J}}$. Hence, $h \in \mathcal{C}_{\mathcal{J}, \mathcal{J}} \subset \mathcal{C}_{\mathcal{J}, \text { nat }}$. Since

$$
h^{-1}\left(\left(-1, \frac{1}{2}\right)\right)=\bigcup_{k \in \mathbb{Z}}\left[k, k+\frac{1}{2}\right) \notin \mathcal{T}_{\text {nat }},
$$

we get that $h \notin \mathcal{C}_{\text {nat }, \text { nat }}$ and condition (ii) is satisfied.

A direct consequence of this proof is the following property.

Property 13. Let $\mathcal{J}$ be a sequence of intervals one-side tending to zero. Then the inclusions

(i) $\mathcal{C}_{\text {nat, nat }} \subset \mathcal{C}_{\mathcal{J}, \text { nat }}$,

(ii) $\mathcal{C}_{\mathcal{J}, \mathcal{J}} \subset \mathcal{C}_{\mathcal{J}, \text { nat }}$

are proper.

The subsequent terminology is needed in the reminder of this section.

Either of the sets

$$
\bigcup_{n \in \mathbb{N}}\left(a_{n}, b_{n}\right), \quad \bigcup_{n \in \mathbb{N}}\left[a_{n}, b_{n}\right]
$$

is a right interval set at a point $x_{0}$ if $x_{0}<b_{n+1}<a_{n}<b_{n}$ for $n \in \mathbb{N}$ and $\lim _{n \rightarrow \infty} a_{n}=x_{0}$.

In the case when $a_{n}<b_{n}<a_{n+1}<x_{0}$ for $n \in \mathbb{N}$ and $\lim _{n \rightarrow \infty} a_{n}=x_{0}$ it is called a left interval set at a point $x_{0}$.

We will call the union of a right interval set and a left interval set at the same point $x_{0}$ a both interval set at a point $x_{0}$. A set $A$ is an interval set at a point $x_{0}$ if it is a right interval or a left interval or a both interval set at a point $x_{0}$. An interval set at a point 0 is simply called an interval set. 
TheOREM 14. For every sequence of intervals $\mathcal{J} \in \Im$ there exists an interval set $B$ consisting of open intervals such that 0 is an $\mathcal{J}$-density point of $B$.

Pro of. Let $J_{n}=\left[a_{n}, b_{n}\right]$ for $n \in \mathbb{N}$ and define

$$
M_{r}=\left\{n \in \mathbb{N}: b_{n}>0\right\}, \quad M_{l}=\left\{n \in \mathbb{N}: a_{n}<0\right\} .
$$

Clearly, at least one of these sets is infinite. There are three possibilities:

$1^{0}$ The set $M_{r}$ is infinite and the set $M_{l}$ is finite. We can assume that the set $M_{l}$ is empty, hence $a_{n} \geq 0$ for $n \in \mathbb{N}$. Let $c_{1}=\frac{1}{2}$ and

$$
\begin{aligned}
I(1) & =\left\{k \in \mathbb{N}: J_{k} \cap\left(\frac{1}{2}, 1\right) \neq \emptyset\right\}, \\
j(1) & =\max (\{1\} \cup\{k \in I(1)\}), \\
z_{1} & =\min \left(\left\{\frac{1}{2}\right\} \cup\left\{\left|J_{k} \cap\left(\frac{1}{2}, 1\right)\right|: k \in I(1)\right\}\right), \\
d_{1} & =c_{1}+2^{-j(1)} z_{1} .
\end{aligned}
$$

If we define $c_{k}, I(k), j(k), z_{k}$ and $d_{k}$ for $k=1,2, \ldots, n-1$, then there exists a natural number $i(n)$ such that $2^{1-i(n)} \leq\left|\left[c_{n-1}, d_{n-1}\right]\right|$. We put $c_{n}=2^{-i(n)}$ and

$$
\begin{aligned}
I(n) & =\left\{k \in \mathbb{N}: J_{k} \cap\left(c_{n}, 2 c_{n}\right) \neq \emptyset\right\}, \\
j(n) & =\max (\{n\} \cup\{k \in I(n)\}), \\
z_{n} & =\min \left(\left\{c_{n}\right\} \cup\left\{\left|J_{k} \cap\left(c_{n}, 2 c_{n}\right)\right|: k \in I(n)\right\}\right), \\
d_{n} & =c_{n}+2^{-j(n)} z_{n} .
\end{aligned}
$$

Notice that

$$
d_{n} \leq 2 c_{n} \leq\left|\left[c_{n-1}, d_{n-1}\right]\right|<c_{n-1}
$$

and

$$
\left|\left[c_{n}, d_{n}\right]\right| \leq 2^{-j(n)}\left|J_{k}\right| \leq 2^{-k}\left|J_{k}\right| \quad \text { for } k \in \mathbb{N}
$$

Putting

$$
B=\bigcup_{n \in \mathbb{N}}\left(d_{n+1}, c_{n}\right),
$$

we obtain that $B$ is a right interval set. Moreover,

$$
\left(J_{n} \backslash B\right) \subset J_{n} \cap\left(\left[0, d_{k(n)+1}\right] \cup\left[c_{k(n)}, d_{k(n)}\right]\right),
$$

where $k(n)=\min \left\{k \in \mathbb{N}: J_{n} \cap\left[c_{k}, d_{k}\right] \neq \emptyset\right\}$ for $n \in \mathbb{N}$. From (3) we obtain

$$
\left|J_{n} \cap\left[c_{k(n)}, d_{k(n)}\right]\right| \leq\left|\left[c_{k(n)}, d_{k(n)}\right]\right| \leq 2^{-n}\left|J_{n}\right| .
$$




\section{JACEK HEJDUK — ANNA LORANTY — RENATA WIERTELAK}

In addition, from (2) and (3) we have

$$
\left|J_{n} \cap\left[0, d_{k(n)+1}\right]\right| \leq d_{k(n)+1} \leq\left|\left[c_{k(n)}, d_{k(n)}\right]\right| \leq 2^{-n}\left|J_{n}\right| .
$$

Hence, for every $n \in \mathbb{N}$ we have

$$
\lambda\left(J_{n} \backslash B\right) \leq 2^{1-n}\left|J_{n}\right| .
$$

Therefore,

$$
\begin{aligned}
\lim _{n \rightarrow \infty} \frac{\lambda\left(B \cap J_{n}\right)}{\left|J_{n}\right|} & =\lim _{n \rightarrow \infty} \frac{\left|J_{n}\right|-\lambda\left(J_{n} \backslash B\right)}{\left|J_{n}\right|} \\
& \geq \lim _{n \rightarrow \infty} \frac{\left|J_{n}\right|-2^{1-n}\left|J_{n}\right|}{\left|J_{n}\right|} \geq \lim _{n \rightarrow \infty} 1-2^{1-n}=1 .
\end{aligned}
$$

For that reason, 0 is an $\mathcal{J}$-density point of $B$.

$2^{0}$ The set $M_{l}$ is infinite and the set $M_{r}$ is finite. In this case we consider sequence of intervals $-\mathcal{J}=\left\{-J_{n}\right\}_{n \in \mathbb{N}}$. From the first part of the proof, there exists a right interval set $C$ consisting of open intervals such that 0 is an $(-\mathcal{J})$ -density point of $C$. Putting $B=-C$, we obtain a left interval set $B$ composed of open intervals such that $0 \in \Phi_{\mathcal{J}}(B)$.

$3^{0}$ Both sets, $M_{l}$ and $M_{r}$, are infinite. Let $M_{l}=\left\{l_{n}: n \in \mathbb{N}\right\}$ and $M_{r}=$ $\left\{r_{n}: n \in \mathbb{N}\right\}$. We then consider sequences of intervals $\mathcal{J}_{L}=\left\{L_{n}\right\}_{n \in \mathbb{N}}$ and $\mathcal{J}_{R}=$ $\left\{R_{n}\right\}_{n \in \mathbb{N}}$, where

$$
\begin{array}{ll}
L_{n}=J_{n} \cap(-\infty, 0] & \text { for } n \in M_{l}, \\
L_{n}=\left(-J_{n}\right) \cap(-\infty, 0] & \text { for } n \notin M_{l}, \\
R_{n}=J_{n} \cap[0, \infty) & \text { for } n \in M_{r}, \\
R_{n}=\left(-J_{n}\right) \cap[0, \infty) & \text { for } n \notin M_{r} .
\end{array}
$$

As in the first part, we define $B_{r}$. It is a right interval set consisting of open intervals such that it is an $\mathcal{J}_{R}$-density point of $B_{r}$. Similarly we define a left interval set $B_{l}$ consisting of open intervals such that 0 is an $\mathcal{J}_{L}$-density point of $B_{l}$. Then, the set $B=B_{l} \cup B_{r}$ is the interval set composed of open intervals. We must show that 0 is an $\mathcal{J}$-density point of $B$.

It follows from (4) that

$$
\lambda\left(L_{n} \backslash B\right) \leq 2^{1-n}\left|L_{n}\right| \quad \text { and } \quad \lambda\left(R_{n} \backslash B\right) \leq 2^{1-n}\left|R_{n}\right| \quad \text { for } \quad n \in \mathbb{N} .
$$

Therefore,

$\lambda\left(J_{n} \backslash B\right) \leq \lambda\left(L_{n} \backslash B\right)+\lambda\left(R_{n} \backslash B\right) \leq 2^{1-n}\left|L_{n}\right|+2^{1-n}\left|R_{n}\right| \leq 2^{2-n}\left|J_{n}\right| \quad$ for $\quad n \in \mathbb{N}$.

It implies that

$$
\lim _{n \rightarrow \infty} \frac{\lambda\left(B \cap J_{n}\right)}{\left|J_{n}\right|} \geq \lim _{n \rightarrow \infty} 1-2^{2-n}=1 .
$$

As a result, $0 \in \Phi_{\mathcal{J}}(B)$. 


\section{ON $\mathcal{J}$-CONTINUOUS FUNCTIONS}

As a simple consequence of the proof of the previous theorem, we obtain the following:

TheOREm 15. For every sequence of intervals $\mathcal{J}$ tending to zero there exists a sequence of intervals $\mathcal{K}$ tending to zero such that topologies generated by $\mathcal{J}$ and $\mathcal{K}$, respectively, are incomparable.

Proof. The set $C=[-1,1] \backslash(B \cup\{0\})$, where $B$ is the set from the previous proof, is an interval set composed of closed intervals. We order them in the sequence $\mathcal{K}$. Then, $0 \in \Phi_{\mathcal{J}}(B), 0 \notin \Phi_{\mathcal{K}}(B), 0 \notin \Phi_{\mathcal{J}}(C), 0 \in \Phi_{\mathcal{K}}(C)$. It is easy to conclude that $\mathcal{T}_{\mathcal{J}} \backslash \mathcal{T}_{\mathcal{K}} \neq \emptyset$ and $\mathcal{T}_{\mathcal{K}} \backslash \mathcal{T}_{\mathcal{J}} \neq \emptyset$.

Property 16. Let $\mathcal{J} \in \Im$ and $A$ be an open interval set such that $0 \in \Phi_{\mathcal{J}}(A)$. Then, there exists an interval set $B \subset A$ composed of closed intervals such that $0 \in \Phi_{\mathcal{J}}(B)$.

Proof. Let $A=\bigcup_{k \in \mathbb{N}} A_{k}$, where $A_{k}$ are disjoint open intervals. Putting

$$
N_{k}:=\left\{i \in \mathbb{N}: J_{i} \cap A_{k} \neq \emptyset\right\} \text { for every } k \in \mathbb{N},
$$

we obtain a finite set. Then we define

$$
\begin{aligned}
j_{k} & :=\max \left(\{k\} \cup\left\{i: i \in N_{k}\right\}\right), \\
l_{k} & :=\min \left(\left\{\left|A_{k}\right|\right\} \cup\left\{\left|J_{i}\right|: i \in N_{k}\right\}\right) .
\end{aligned}
$$

Let $B_{k}$ be a closed subinterval of $A_{k}$ such that $\lambda\left(A_{k} \backslash B_{k}\right) \leq 2^{-\left(k+j_{k}\right)} l_{k}$ for $k \in \mathbb{N}$. Observe that for any $n \in \mathbb{N}$ and $k \in \mathbb{N}$ we have

$$
\begin{aligned}
\lambda\left(B_{k} \cap J_{n}\right) & =\lambda\left(A_{k} \cap J_{n}\right)-\lambda\left(\left(A_{k} \backslash B_{k}\right) \cap J_{n}\right) \\
& \geq \lambda\left(A_{k} \cap J_{n}\right)-2^{-\left(k+j_{k}\right)} l_{k} \\
& \geq \lambda\left(A_{k} \cap J_{n}\right)-2^{-(k+n)}\left|J_{n}\right| .
\end{aligned}
$$

Hence, the following holds for $B=\bigcup_{k \in \mathbb{N}} B_{k}$ and any $n \in \mathbb{N}$ :

$$
\begin{aligned}
\lambda\left(B \cap J_{n}\right) & =\lambda\left(\bigcup_{k \in \mathbb{N}} B_{k} \cap J_{n}\right)=\sum_{k \in \mathbb{N}} \lambda\left(B_{k} \cap J_{n}\right) \\
& \geq \sum_{k \in \mathbb{N}}\left(\lambda\left(A_{k} \cap J_{n}\right)-2^{-(k+n)}\left|J_{n}\right|\right) \\
& =\sum_{k \in \mathbb{N}} \lambda\left(A_{k} \cap J_{n}\right)-\sum_{k \in \mathbb{N}} 2^{-(k+n)}\left|J_{n}\right|=\lambda\left(A \cap J_{n}\right)-2^{-n}\left|J_{n}\right| .
\end{aligned}
$$

Therefore,

$$
\lim _{n \rightarrow \infty} \frac{\lambda\left(B \cap J_{n}\right)}{\left|J_{n}\right|} \geq \lim _{n \rightarrow \infty} \frac{\lambda\left(A \cap J_{n}\right)-2^{-n}\left|J_{n}\right|}{\left|J_{n}\right|}=\lim _{n \rightarrow \infty} \frac{\lambda\left(A \cap J_{n}\right)}{\left|J_{n}\right|}-2^{-n} .
$$

Since $0 \in \Phi_{\mathcal{J}}(A)$, we have $0 \in \Phi_{\mathcal{J}}(B)$. 


\section{JACEK HEJDUK — ANNA LORANTY — RENATA WIERTELAK}

Property 17. Let $\mathcal{J} \in \Im$. Then, there exists $\mathcal{K} \in \Im$ such that $\mathcal{T}_{\mathcal{J}} \neq \mathcal{T}_{\mathcal{K}}$, $\mathcal{C}_{\mathcal{J}, \mathcal{J}} \neq \mathcal{C}_{\mathcal{K}, \mathcal{K}}$ and $\mathcal{C}_{\mathcal{J}, \text { nat }} \neq \mathcal{C}_{\mathcal{K}, \text { nat }}$.

P r o of. $1^{o}$ Suppose that the sequence $\mathcal{J}$ is left-side tending to zero. Then, as a sequence $\mathcal{K}$, we put any sequence right-side tending to zero and we define the function $f: \mathbb{R} \rightarrow \mathbb{R}$ in the following way: $f(x)=\chi_{[0, \infty)}$. Obviously, Theorem 9 implies that $\mathcal{T}_{\mathcal{J}} \neq \mathcal{T}_{\mathcal{K}}$. Moreover, it is easy to see that $f \notin \mathcal{C}_{\mathcal{J}, \text { nat }}$. So, we obtain that

$$
f \in \mathcal{C}_{\mathcal{K}, \mathcal{K}} \subset \mathcal{C}_{\mathcal{K}, \text { nat }} \quad \text { and } \quad f \notin \mathcal{C}_{\mathcal{J}, \text { nat }} \supset \mathcal{C}_{\mathcal{J}, \mathcal{J}}
$$

$2^{o}$ Suppose that the sequence $\mathcal{J}$ is not left-side tending to zero. Then, as a sequence $\mathcal{K}$, we put any sequence left-side tending to zero and we define function $f: \mathbb{R} \rightarrow \mathbb{R}$ in the following way: $f(x)=\chi_{(0, \infty)}$. Arguments similar to those above show that

$$
f \in \mathcal{C}_{\mathcal{K}, \mathcal{K}} \subset \mathcal{C}_{\mathcal{K}, \text { nat }} \quad \text { and } \quad f \notin \mathcal{C}_{\mathcal{J}, \text { nat }} \supset \mathcal{C}_{\mathcal{J}, \mathcal{J}}
$$

Let $\mathcal{J}=\left\{J_{n}\right\}_{n \in \mathbb{N}}$ and $\mathcal{K}=\left\{K_{n}\right\}_{n \in \mathbb{N}}$ be sequences of intervals. Then, the sequence ordered in an arbitrary fashion containing all intervals of the sequences $\mathcal{J}$ and $\mathcal{K}$, and denoted by $\mathcal{J} \cup \mathcal{K}$, is called the union of sequences $\mathcal{J}$ and $\mathcal{K}$.

Property 18. The sequences of intervals $\mathcal{J}$ and $\mathcal{K}$ are tending to zero if and only if the sequence $\mathcal{J} \cup \mathcal{K}$ is tending to zero.

Property 19. If $\mathcal{J} \in \Im$ and $\mathcal{K} \in \Im$, then

$$
\mathcal{T}_{\mathcal{J} \cup \mathcal{K}}=\mathcal{T}_{\mathcal{J}} \cap \mathcal{T}_{\mathcal{K}}
$$

P r o of. It is a direct consequence of the following fact:

$$
\Phi_{\mathcal{J} \cup \mathcal{K}}(A)=\Phi_{\mathcal{J}}(A) \cap \Phi_{\mathcal{K}}(A) .
$$

Property 20. Let $\mathcal{J} \in \Im$ and $\mathcal{K} \in \Im$. Then,

(i) $\mathcal{C}_{\mathcal{J}, \text { nat }} \cap \mathcal{C}_{\mathcal{K}, \text { nat }}=\mathcal{C}_{\mathcal{J} \cup \mathcal{K}, \text { nat }}$,

(ii) $\mathcal{C}_{\text {nat, } \mathcal{J}} \cap \mathcal{C}_{\text {nat }, \mathcal{K}}=\mathcal{C}_{\text {nat }, \mathcal{J} \cup \mathcal{K} \text {, }}$

(iii) $\mathcal{C}_{\mathcal{J}, \mathcal{J}} \cap \mathcal{C}_{\mathcal{K}, \mathcal{K}} \subsetneq \mathcal{C}_{\mathcal{J} \cup \mathcal{K}, \mathcal{J} \cup \mathcal{K}}$

Proof. The condition (i) and the inclusion (iii) are evident by Property 19. The condition (ii) follows from Property 10, Let

$$
\mathcal{J}=\left\{\left[-\frac{1}{n}, 0\right]\right\}_{n \in \mathbb{N}}, \quad \mathcal{K}=\left\{\left[0, \frac{1}{n}\right]\right\}_{n \in \mathbb{N}} .
$$

Then, $\mathcal{J}$ is left-side tending to zero and $\mathcal{K}$ is right-side tending to zero. It is easy to observe that $\mathcal{T}_{\mathcal{J} \cup \mathcal{K}}$ is the density topology. Thus, the function $f(x)=-x$ 


\section{ON $\mathcal{J}$-CONTINUOUS FUNCTIONS}

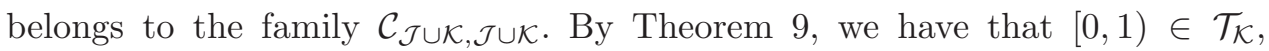
whereas $f^{-1}([0,1))=(-1,0] \notin \mathcal{T}_{\mathcal{K}}$. It implies that

$$
f \notin \mathcal{C}_{\mathcal{K}, \mathcal{K}} \quad \text { so } \quad f \notin\left(\mathcal{C}_{\mathcal{J}, \mathcal{J}} \cap \mathcal{C}_{\mathcal{K}, \mathcal{K}}\right) .
$$

\section{REFERENCES}

[1] BRUCKNER, A. M.: Differentiation of Real Functions. Lecture Notes in Math., Vol. 659, Springer Verlag, Berlin, 1978.

[2] CSÖRNYEI, M.: Density theorems revisited, Acta Sci. Math. 64 (1998), 59-65.

[3] FILIPCZAK, M.-FILIPCZAK, T.-HEJDUK, J.: On the comparison of the density type topologies, Atti Semin. Mat. Fis. Univ. Modena LII (2004), 1-11.

[4] HEJDUK, J.-WIERTELAK, R.: On the Abstract Density Topologies Generated by Lower and Almost Lower Density Operators. Traditional and Present-day Topics in Real Analysis, Łodź University Press, 2013.

[5] HEJDUK, J.-WIERTELAK, R.: On the generalization of density topologies on the real line, Math. Slovaca 64 (2014), 1267-1276.

[6] WILCZYŃSKI, W.: Density topologies, in: Handbook of Measure Theory (E. Pap., ed.), North Holland, Amsterdam, 2002, pp. 675-702.

Received December 3, 2014
Łódź University

Faculty of Mathematics

and Computer Science

Banacha 22

PL-90-238 Eódź

POLAND

E-mail: hejduk@math.uni.lodz.pl loranta@math.uni.lodz.pl wiertelak@math.uni.lodz.pl 